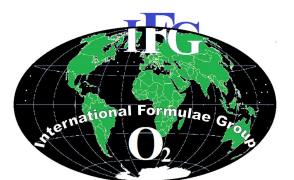

Available online at http://www.ifgdg.org

Int. J. Biol. Chem. Sci. 10(4): 1534-1541, August 2016

International Joumal

of Biological and

Chemical Sciences

ISSN 1997-342X (Online), ISSN 1991-8631 (Print)

Original Paper http://ajol.info/index.php/ijbcs http://indexmedicus.afro.who.int

\title{
Plantes médicinales utilisées dans le traitement traditionnel du paludisme à Bamako (Mali)
}

\author{
Mamadou Lamine DIARRA ${ }^{1 *}$, Madani MARIKO ${ }^{2}$, Mame Samba MBAYE ${ }^{1}$ et \\ Kandioura NOBA ${ }^{1}$ \\ ${ }^{1}$ Laboratoire de Botanique et Biodiversité, Université Cheikh Anta Diop de Dakar, B.P.5005, Dakar Fann, \\ Sénégal. \\ ${ }^{2}$ Laboratoire de Chimie Analytique, Faculté de Pharmacie, Université des Sciences, des techniques et des \\ Technologies de Bamako, B.P. 1805, Mali. \\ *Auteur correspondant ; E-mail : mldiarra.edu.ml@gmail.com
}

\section{RESUME}

Le paludisme constitue un problème de santé publique à Bamako et l'utilisation des plantes dans sa prise en charge est fréquente. Cependant beaucoup de menaces pèsent sur ces plantes à cause de plusieurs facteurs dont leur méconnaissance d'où la nécessité de les connaitre pour améliorer leur gestion et leur conservation. Une enquête ethnobotanique a été conduite à Bamako d'Août 2012 à Novembre 2012. Les résultats ont montré que 52 espèces reparties dans 48 genres, 24 familles sont utilisées. Les familles les plus représentées étaient les Caesalpiniaceae, les Rubiaceae, les Combretaceae, les Euphorbiaceae et les Fabaceae. Cette flore était dominée surtout par les espèces africaines et pantropicales. Le type biologique le plus nombreux était constitué par les phanérophytes. Les feuilles constituaient la partie la plus utilisée, la préparation la plus utilisée était la décoction. Les résultats de cette étude pourront ainsi contribuer à la connaissance exhaustive des plantes à visée antipaludique et à une valorisation de la médecine traditionnelle Malienne.

(C) 2016 International Formulae Group. All rights reserved.

Mots clés : Paludisme, plantes médicinales, ethnobotanique, Bamako.

\section{Medicinal plants used in traditional treatment of malaria in Bamako (Mali)}

\section{ABSTRACT}

Malaria is a public health problem in Bamako and the use of plants in its management is common. However many threats to these plants were observed because of several factors including their lack of the need to know them to improve their management and conservation. An ethnobotanical survey was conducted in Bamako from August 2012 to November 2012. The results showed that 52 species distributed in 48 genera, 24 families are used. The most represented families were Caesalpiniaceae, Rubiaceae, the Combretaceae, 
Euphorbiaceae and Fabaceae. This flora was dominated mostly by African and pan-tropical species. The largest biological type was made by phanerophytes. The leaves are the most used part of the most common preparation was the decoction. The results of this study could contribute to the comprehensive knowledge of plants with antimalarial properties and valorization of traditional Malian medicine.

(C) 2016 International Formulae Group. All rights reserved.

Keywords: Malaria, medicinal plants, ethnobotany, Bamako.

\section{INTRODUCTION}

Le paludisme, est une maladie infectieuse très rependu avec 219 millions de cas en 2010 et 660000 décès soit une diminution de la mortalité de $25 \%$ au niveau mondial par rapport à 2000 et de $33 \%$ dans la région africaine de l'OMS, la plupart des décès surviennent chez des enfants vivant en Afrique, où chaque minute un enfant meurt du paludisme ( Grellier et al., 2008 ; Zhang et al., 2014).

Au Mali, il est la principale cause de mortalité (13\%), de morbidité $(15,6 \%)$ au sein de la population générale, responsable de la plupart des faibles poids de naissance et représente la première cause de décès chez les enfants de moins de cinq (5) ans (Ministère de la Santé du Mali, 2005 ; Djimde et al., 2015). Le paludisme constitue et demeure un problème de santé publique au Mali (Daffe, 2005).

Ainsi la ville de Bamako est caractérisée par une endémicité stable marquée par une recrudescence saisonnière pendant et après la saison des pluies, c'est-àdire de Juin à Décembre, avec une létalité relativement élevée, notamment chez les enfants (Sangare, 2009).

$\mathrm{Au}$ Mali, l'itinéraire thérapeutique montre que la famille et les tradithérapeutes constituent le premier recours de plus de $80 \%$ de la population. Les structures de santé sont consultées dans la majorité des cas après passage des malades chez un tradithérapeute (Diallo et Dussart, 2008). En effet au Mali comme la plupart des pays en développement à cause de la pauvreté, le premier recours en matière de traitement du paludisme est l'utilisation des plantes médicinales (Amuamuta et Na-Bangchang, 2015; Ramadhani et al., 2015). Il s'impose alors le besoin urgent de valoriser cette pratique pour une meilleure gestion et conservation des espèces utilisées. Ainsi cette étude vise à connaitre les plantes utilisées par la population de Bamako pour le traitement traditionnel du paludisme.

\section{MATERIEL ET METHODES}

Une étude transversale a été réalisée à Bamako dont les coordonnées géographiques sont précisées sur les Figures 1 et 2. Le matériel était constitué des fiches de questionnaire, de la flore du Sénégal et de la flore tropicale d'Afrique de l'Ouest (Flora of West Tropical Africa 1954, 1963 et 1968).

La méthode utilisée consistait à mener une enquête ethnobotanique au près de la population d'étude. Pour ce faire, un questionnaire a été utilisé pour la collecte des données auprès des personnes ciblées. Au total, 100 personnes ont été interrogées sur le paludisme et les plantes qu'ils utilisent dans le traitement traditionnel. Le Bamanankan (langue locale du Mali) et le Français ont été utilisés pour l'administration du questionnaire. Chaque personne a été interrogée individuellement.

La nomenclature scientifique employée est celle de Lebrun et Stork (Arbonnier, 2009). Les données ont été analysées avec le tableur Excel 2007 et Epi info version 3.5. 
- Latitude: $12^{\circ} 37^{\prime} \mathrm{N}$.

- Longitude: $8^{\circ} 1^{\prime} \mathrm{W}$

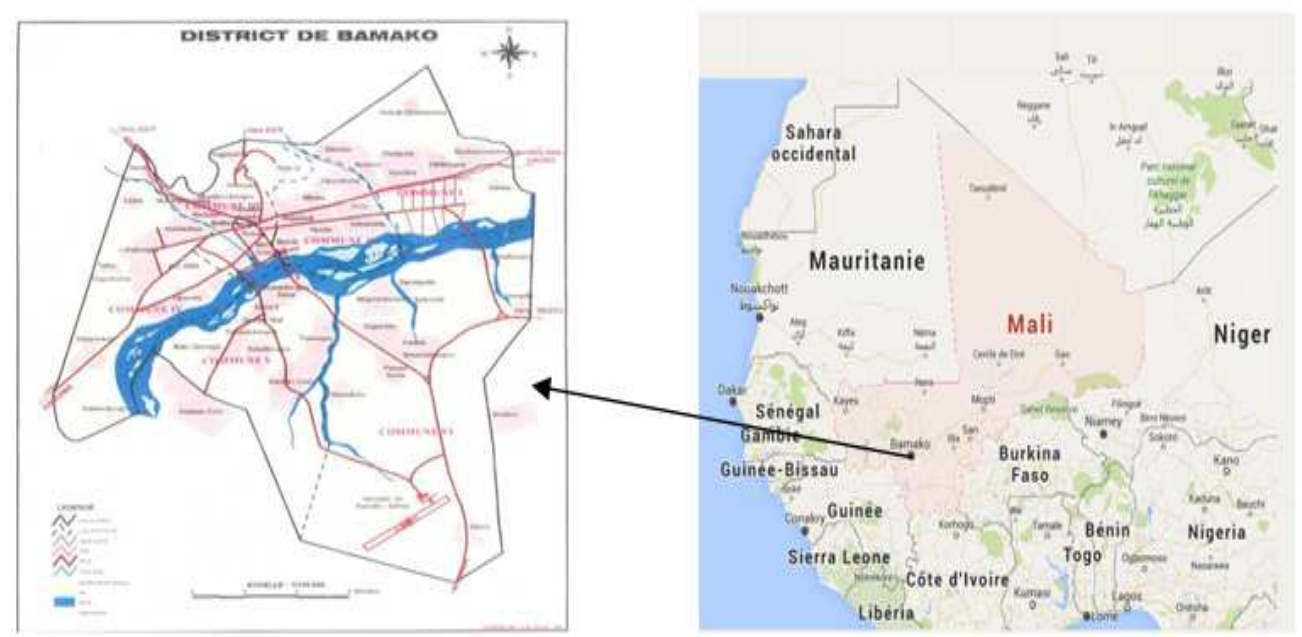

Figure 2: Carte administrative de Bamako

Figure 1: Carte du Mali

(Institut géographique du Mali, 2009)

\section{RESULTATS}

Les résultats de l'enquête ont montré que cinquante deux (52) espèces, reparties dans 49 genres et 24 familles sont utilisées pour le traitement traditionnel du paludisme. Ces espèces sont africaines, pantropicales, afro-asiatiques, afro-malgaches, asiatiques, australiennes, américaines, cosmopolites et Européennes. L'ethnie Bambara représentait plus de la majorité des personnes enquêtées. Les types biologiques rencontrés sont les
Phanérophytes, les Thérophytes, les Géophytes, les Hydrophytes et les Chaméphytes. Les organes utilisés sont les feuilles $(78,4 \%)$, les racines $(13,3 \%)$, les tiges $(5 \%)$ et les fruits $(3,3 \%)$. Les préparations utilisées sont la décoction, la macération, la forme poudre et le fruit cru. Une seule contre indication a été signalée à savoir Cassia alata qui serait déconseillée en cas de diarrhée. Ces informations sont récapitulées dans le Tableau 1. 
M. L. DIARRA et al. / Int. J. Biol. Chem. Sci. 10(4): 1534-1541, 2016

Tableau 1: Liste des espèces recensées à Bamako pour le traitement du paludisme avec les types biologiques, les affinités biogéographiques, les organes et les préparations utilisés.

\begin{tabular}{|c|c|c|c|c|c|}
\hline Familles & Espèces & Types biologiques & $\begin{array}{l}\text { Affinités } \\
\text { biogéographiques }\end{array}$ & Organes utilisés & $\begin{array}{r}\text { Préparations } \\
\text { des recettes }\end{array}$ \\
\hline \multirow[t]{2}{*}{ Anacardiaceae } & Sclerocarya birrea (A.Rich.) Hochst & $\mathrm{P}$ & Af & $\mathrm{Fe}$ & Déc \\
\hline & Mangifera indica $\mathrm{L}$. & $\mathrm{P}$ & $\mathrm{Pt}$ & $\mathrm{Fe}, \mathrm{Ec}$ du tronc & Déc \\
\hline Annonaceae & Annona senegalensis Pers. & $\mathrm{P}$ & Af & $\mathrm{Rac}$ & Déc \\
\hline \multirow[t]{2}{*}{ Arecaceae } & Raphia sudanica A.Chev & $\mathrm{P}$ & Af & $\mathrm{Fe}$ & Déc \\
\hline & Ethulia conyzoides $\mathrm{L}$. & $\mathrm{T}$ & As & $\mathrm{Fe}$ & Déc \\
\hline Asteraceae & Centaurea perrottettii DC. & $\mathrm{T}$ & Af & $\mathrm{Fe}$ & Déc \\
\hline Bignoniaceae & Crescentia cujete L. & $\mathrm{P}$ & Am & $\mathrm{Fe}$ & Déc \\
\hline Cactaceae & Carica papaya $\mathrm{L}$. & $\mathrm{P}$ & $\mathrm{Pt}$ & $\mathrm{Fe}$ & Déc \\
\hline \multirow[t]{7}{*}{ Caesalpiniaceae } & Cassia alata (L.) Roxb & $\mathrm{T}$ & $\mathrm{Pt}$ & Fruit & Déc \\
\hline & Cassia occidentalis $\mathrm{L}$. & $\mathrm{T}$ & $\mathrm{Pt}$ & $\mathrm{Fe}$ & Déc \\
\hline & Cassia siamea (Lam.)Irwin \& Barneby & $\mathrm{P}$ & $\mathrm{Pt}$ & $\mathrm{Fe}$ & Déc \\
\hline & Cassia sieberiana DC. & $\mathrm{P}$ & Af & Fe et Rac & Déc \\
\hline & Daniellia olivieri (Rolfe) Hutch \& Dalz. & $\mathrm{P}$ & Af & $\mathrm{Fe}$ & Déc \\
\hline & Delonix regia (Boj.) Raf. & $\mathrm{P}$ & M & $\mathrm{Fe}$ & Déc \\
\hline & Piliostigma thonningii (Schumach.) Milne-Redh. & $\mathrm{P}$ & Af & $\mathrm{Fe}$ & Déc \\
\hline Cochlospermaceae & Cochlospermum tinctorium A. Rich, Gull \& Perr & G & Af & $\mathrm{Fe}$ & Déc \\
\hline \multirow[t]{2}{*}{ Combretaceae } & Anogeissus leiocarpus (DC.) Gull \& Perr & $\mathrm{P}$ & Af & $\mathrm{Fe}, \mathrm{Ec}$ du tronc et Rac & Déc \\
\hline & Combretum glutinosum Perr. \& DC. & $\mathrm{P}$ & Af & $\mathrm{Fe}$ & Déc \\
\hline
\end{tabular}


M. L. DIARRA et al. / Int. J. Biol. Chem. Sci. 10(4): 1534-1541, 2016

\begin{tabular}{|c|c|c|c|c|c|}
\hline & Combretum micranthum G. Don & $\mathrm{P}$ & Af & $\mathrm{Fe}$ & Déc \\
\hline & Guiera senegalensis G.F. Gmel. & $\mathrm{P}$ & Af & $\mathrm{Fe}$ & Déc \\
\hline & Pteleopsis suberosa Engl. \& Diels & $\mathrm{P}$ & Af & $\mathrm{Fe}$ & Déc \\
\hline Convolvulaceae & Ipomoea asarifolia (Desr.) Roem. \& Schult. & $\mathrm{T}$ & AmAs & $\mathrm{Fe}$ & Déc \\
\hline \multirow[t]{6}{*}{ Euphorbiaceae } & Hymenocardia acida Tul. & $\mathrm{P}$ & Af & $\mathrm{Fe}$ & Déc \\
\hline & Jatropha curcas L. & $\mathrm{P}$ & $\mathrm{Pt}$ & $\mathrm{Fe}$ & Déc \\
\hline & Phyllanthus reticulatus Poir. & $\mathrm{P}$ & Af & $\mathrm{Fe}$ & Déc \\
\hline & Ricinus communis $\mathrm{L}$. & $\mathrm{P}$ & $\mathrm{Pt}$ & $\mathrm{Fe}$ & Déc \\
\hline & Securinega virosa (Roxb. ex.Willd) Voigt & $\mathrm{P}$ & Asu & $\mathrm{Fe}$ & Déc \\
\hline & Afrormosia laxiflora (Benth ex.Bak) Harms & $\mathrm{P}$ & Af & $\mathrm{Fe}$ & Déc \\
\hline \multirow[t]{3}{*}{ Fabaceae } & Erithrina senegalensis DC. & $\mathrm{P}$ & Af & $\mathrm{Fe}$ & Déc \\
\hline & Indigofera tinctoria $\mathrm{L}$. & $\mathrm{T}$ & As & $\mathrm{Fe}$ & Déc \\
\hline & Stylosanthes mucronata Willd. & $\mathrm{T}$ & Mas & $\mathrm{Fe}$ & Déc \\
\hline Lamiaceae & Ocimum basilicum $\mathrm{L}$. & $\mathrm{C}$ & Cosm & $\mathrm{Fe}$ & Déc \\
\hline Malvaceae & Sida acuta Burm. & $\mathrm{T}$ & AmAs & $\mathrm{Fe}$ & Déc \\
\hline & Trichilia emetica Vahl & $\mathrm{P}$ & Mas & $\mathrm{Fe}$ & Déc \\
\hline \multirow[t]{2}{*}{ Mimosaceae } & Dichrostachys glomerata (Forsk.) Chiov. & $\mathrm{P}$ & Af & $\mathrm{Fe}$ & Déc \\
\hline & Entada africana Guill \& Perr. & $\mathrm{P}$ & Af & Fe et $\mathrm{Rac}$ & Déc et Mac \\
\hline Musaceae & Musa acuminata Colla & $\mathrm{P}$ & $\mathrm{Pt}$ & $\mathrm{Fe}$ & Déc \\
\hline Myrtaceae & Eucalyptus camaldulensis Dehnh & $\mathrm{P}$ & $\mathrm{Pt}$ & $\mathrm{Fe}$ & Déc \\
\hline Nymphaeaceae & Nymphaea lotus $\mathrm{L}$. & Hy & Mase & $\mathrm{Fe}$ & Déc \\
\hline Opiliaceae & Opilia celtidifolia (Guill. \& Perr) Endl. Ex Walp. & $\mathrm{P}$ & Af & $\mathrm{Fe}$ & Déc \\
\hline
\end{tabular}


M. L. DIARRA et al. / Int. J. Biol. Chem. Sci. 10(4): 1534-1541, 2016

\begin{tabular}{|c|c|c|c|c|c|}
\hline \multirow[t]{2}{*}{ Poaceae } & Oxytenanthera abyssinica (A.Rich.) Munro & $\mathrm{P}$ & Af & $\mathrm{Fe}$ & Déc \\
\hline & Pennisetum pedicellatum Trin. & $\mathrm{T}$ & As & $\mathrm{Fe}$ & Déc \\
\hline \multirow[t]{7}{*}{ Rubiaceae } & Canthium schimperianum A. Rich. & $\mathrm{P}$ & Af & $\mathrm{Fe}$ & Déc \\
\hline & Feretia apodenthera Del. & $\mathrm{P}$ & Af & $\mathrm{Fe}$ & Déc \\
\hline & Crossopterix febrifuga (Afzel. ex G. Don) Benth. & $\mathrm{P}$ & Af & $\mathrm{Fe}$ & Déc \\
\hline & Gardenia sokotensis Hutch & $\mathrm{P}$ & Af & $\mathrm{Fe}$ & Déc \\
\hline & Macrosphyra longistyla (DC.) Hiern & $\mathrm{P}$ & Af & $\mathrm{Fe}$ & Déc \\
\hline & Mitragyna inermis (Willd) kuntze & $\mathrm{P}$ & Af & $\mathrm{Fe}, \mathrm{Ec}$ du tronc et Rac & Déc et Mac \\
\hline & Nauclea latifolia (Smith) Bruce & $\mathrm{P}$ & Af & Fe et $\mathrm{Rac}$ & Déc et Mac \\
\hline Rutaceae & Citrus limon (L.) Burm.f. & $\mathrm{P}$ & As & $\mathrm{Fe}$ & Déc \\
\hline Solanaceae & Solanum aethiopicum $\mathrm{L}$. & $\mathrm{T}$ & Af & Fruit & Fruit cru \\
\hline
\end{tabular}




\section{DISCUSSION}

Cinq familles regroupent plus de la moitié des espèces recensées : les Rubiaceae et les Caesalpiniaceae ont été signalées avec des pourcentages similaires $(13,47 \%)$; les Euphorbiaceae et les Combretaceae ont eu chacune $9,62 \%$ des citations et les Fabaceae 7,69\%. Le Mitragyna inermis est l'espèce la plus citée avec $40 \%$, suivie par le Combretum micranthum avec $33 \%$ de citations.

Parmi les 52 espèces recensées, les espèces africaines sont les plus signalées avec $57,69 \%$, les Phanérophytes avec $75 \%$ constituaient le type biologique le plus important. Les feuilles constituaient l'organe le plus utilisé avec $78,4 \%$; ce résultat est en conformité avec ceux de Diarra (2003) et Betti et al. (2013). Cet état de fait est très important non seulement pour la survie des plantes car c'est l'organe qui se renouvelle facilement, mais aussi abondant en hivernage pour la majorité des espèces qui correspond à la période ou le taux d'infection est plus élevé (Sangaré, 2009). La décoction représentait la préparation la plus utilisée, soulignée aussi par Betti et al. (2013); Amuamuta et NaBangchang (2015).

\section{Conclusion}

Les plantes utilisées pour le traitement traditionnel du paludisme à Bamako comptent 52 espèces réparties dans 48 genres et 24 familles. Cinq familles représentent plus de la moitié des espèces recensées soit $53,87 \%$. Ces plantes sont caractérisées par une forte présence d'espèces africaines et pantropicales. Cependant deux espèces sont plus utilisées dans le traitement traditionnel du paludisme à savoir le Mitragyna inermis et le Combretum micranthum soit seules, soit le plus souvent en association avec d'autres espèces, surtout en décoction.

Malgré l'abondance des structures sanitaires, les plantes continuent à être utilisées dans le traitement traditionnel du paludisme à Bamako. Il était nécessaire de faire l'état de lieu des différentes espèces de plantes utilisées dans le traitement traditionnel du paludisme, afin d'entreprendre des mesures pour mieux gérer ou protéger celles qui sont menacées.

\section{CONFLIT D'INTERETS}

Il n'y a pas eu de conflit d'intérêts.

\section{CONTRIBUTIONS DES AUTEURS}

MLD a conçu, conduit l'étude et participé à la rédaction de l'article. MSM a participé à la rédaction et à la correction de l'article. $\mathrm{MM}$ et $\mathrm{KN}$ ont relu et corrigé l'article.

\section{REMERCIEMENTS}

Les auteurs remercient le personnel du laboratoire de Botanique et Biodiversité, de l'herbier Dakar du Département de Biologie Végétale de la Faculté des Sciences et Technique de l'UCAD.

\section{REFERENCES}

Amuamuta A, Na-Bangchang K. 2015. A review of ethnopharmacology of the commonly used antimalarial herbal agents for traditional medicine practice in Ethiopia. Afr. J. Pharm. Pharmacol., 9(25): 615-627.

Arbonnier M. 2009. Arbres, Arbustes et Lianes des Zones Sèches d'Afrique de l'Ouest ( ${ }^{\text {ième }}$ éd.). Quae : Paris, p. 573.

Betti JL, Iponga DM, Yongo OD, Mbomio DO, Yobo CM, Ngoy A. 2013. Ethnobotanical study of medicinal plants of the Ipassa-Makokou Biosphere Reserve, Gabon: Plants used for treating malaria. J. Med. Plants Res., 7(31): 2300-2318.

Daffe S. 2005. Connaissances, attitudes et pratiques des populations face au paludisme dans l'aire de santé de Finkolo (Sikasso) Mali. Thèse Médecine, FMPOS, Université Bamako, Bamako, p. 67.

Diallo S, Dussart C. 2008. Importance de la médecine traditionnelle au Mali. Santé Décision Management, 11(1) : 57-64. 
Diarra MN. 2003. Etude phytochimique d'une plante antipaludique utilisée au Mali :Spilanthes oleraceae Jacq (Asteraceae). Thèse Pharmacie, FMPOS, Université Bamako, Bamako, p.78.

Djimde AA, Maiga AW, Ouologuem D, Fofana B, Sagara I, Dembele D, Toure S, Sanogo K, Dama S, Sidibe B, Doumbo OK. 2016. Gametocyte clearance dynamics following oral artesunate treatment of uncomplicated falciparum malaria in Malian children. Parasite, 23(3):1-8.

Grellier P, Depoix D, Schrével J, Florent I. 2008. Discovery of new targets for antimalarial chemotherapy. Parasite, 15: 219-225.

Ministère de la Santé du Mali. 2005. Manuel de formation pour la prise en charge des cas de paludisme au niveau des formations sanitaires. Bamako, p. 58.

Ramadhani SON, Zofou D, Moshi JM, Erasto P, Wanji S, Ngemenya MN, Titanji VPK, Kidukuli AW, Masimba PJ. 2015. Ethnobotanical survey and in vitro antiplasmodial activity of medicinal plants used to treat malaria in Kagera and Lindi regions, Tanzania. J. Med. Plants Res., 9(6):179-192.

Sangare E. 2009. Evaluation de la qualité de la prescription et de la dispensation des CTA dans le district de Bamako. Thèse Pharmacie. FMPOS, Université Bamako, Bamako, p. 115.

Zhang Y, Jiang N, Chang Z, Wang H, Lu H, Wahlgren M, Chen Q. 2014. The var3 genes of Plasmodium falciparum 3D7 strain are differentially expressed in infected erythrocytes. Parasite, 21(19): $1-7$. 